\title{
Modeling and Characterization of in-Body Antennas
}

\author{
Denys Nikolayev ${ }^{1,2}$ \\ ${ }^{1}$ Imec - Ghent University, Ghent, Belgium \\ ${ }^{2}$ Institute of Electronics and Telecommunications of Rennes (IETR), Rennes, France
}

d@deniq.com

\begin{abstract}
Emerging wireless in-body devices pave the way to many breakthroughs in healthcare and clinical research. This technology enables monitoring of physiological parameters while maintaining mobility and freedom of movement of its user. However, establishing reliable communication between an in-body device and external equipment is still a major challenge. The radiation efficiency is constrained by attenuation and reflection losses in tissues. Furthermore, the antennas suffer from impedance detuning issues caused by uncertain electromagnetic properties of body tissues. First, we show that choosing an optimal operating frequency depends on application scenarios and can reduce the losses. Specific designs are then discussed to mitigate the antenna detuning effects due to surrounding biological tissue. Modeling approaches are proposed to lessen the design and optimization complexity. Finally, we present an accurate characterization method of in-body antennas in canonical phantoms using analog fiber optic links.
\end{abstract}

Keywords-biomedical telemetry, implantable, in-body, ingestible, ISM (industrial, scientific, and medical) band, microstrip antennas, miniature antennas, phantom, robust antennas.

\section{INTRODUCTION}

Body-implanted devices can monitor physiological parameters, perform treatments, or establish neural interfaces while maintaining mobility and quality of life of a patient [1]. The devices rely on antennas to transmit biotelemetry data or to receive operational and treatment instructions [2][5]. Multi-band antennas can integrate both data transmission and wireless power transfer functionality increasing the available space inside an in-body device [6]. Design and characterization of such in-body antennas remains challenging as the antenna model must take into account various effects of surrounding tissues on antenna impedance and radiation [7], [8].

To date, establishing a robust medium-range (i.e. $\geqslant 3 \mathrm{~m}$ ) link between an in-body device and external equipment remains a major challenge due to low radiation efficiencies $(\eta<0.1 \%)$ of in-body antennas operating in lossy media [7]. In addition, the antenna impedance is prone to detuning because of uncertain electromagnetic (EM) properties of surrounding tissues [8]. Considering typical maximum input power levels ( $P_{\text {in }} \lesssim 50 \mathrm{~mW}$, limited by safety standards) and receiver (Rx) sensitivities, this ensures a line-of-sight operating range only up to about a few meters. Furthermore, even an intrinsically isotropic in-body antenna obtains directive properties once inside a body due to its radiation not only attenuating but also diffracting and scattering while propagating through highly heterogeneous human body. Accurate human body and antenna modeling makes it possible to study the effects of tissues on the antenna performances and finding ways to improve it.
In this study, we describe approaches to in-body antenna design, modeling, and characterization. The proposed modeling approach ensures the rapid development and robust operation of antennas in a given range of tissues. The antenna performance is assessed in terms of its impedance stability and radiation characteristics in realistic application scenarios. The proposed characterization approach allows for decoupling the antenna from the feeding cable, and the use of a spherical phantom makes it possible to characterize the radiation performance under controlled isotropic conditions.

The paper is organized as follows. Section II describes the biological tissue environment, defines the optimal operating frequency depending on a given in-body application, and overviews the existing approaches to inbody antenna design. Antenna modeling is presented and discussed in Section III. Section IV describes the radiation characterization to validate the numerical results. The paper concludes in Section V.

\section{THEORETICAL CONSIDERATIONS}

\section{A. Electromagnetic Spectrum of Biological Tissue}

Body tissues are an anisotropic, nonlinear, heterogeneous, and dispersive medium [9]. Considering operating frequencies and related power levels, we assume here that the EM properties are isotropic (complex permittivity $\hat{\varepsilon}$ is scalar), nonmagnetic (permeability $\mu=\mu_{0}$ ), and linear due to low supplied power.

The EM spectrum of the tissue is characterized by three main relaxation regions accounting for $(\boldsymbol{\alpha})$ ionic diffusion and membrane-related mechanisms, ( $\boldsymbol{\beta})$ capacitive charging of cellular membranes and intercellular bodies, and $(\gamma)$ dipolar polarization of free water in tissues. A fourth minor dispersion $(\boldsymbol{\delta})$ can also appear in some protein solutions; the mechanisms causing $\boldsymbol{\delta}$ dispersion are the least studied. These relaxation regions define the dispersive EM properties of tissues $\varepsilon(\omega)=\varepsilon^{\prime}(\omega)-j \varepsilon^{\prime \prime}(\omega)=\varepsilon^{\prime}(\omega)-j \sigma(\omega) /\left(\omega \varepsilon_{0}\right)$, where $\varepsilon^{\prime}=$ $\varepsilon_{\mathrm{r}}(\mathbf{r}, \omega) \varepsilon_{0}$ is the permittivity, $\sigma(\mathbf{r}, \omega)$ is the electrical conductivity, $\mathbf{r}$ is the position vector, $\omega=2 \pi f$ is the angular frequency, and $f$ is the frequency.

The EM spectrum of tissues may be accurately modeled using multiple Cole-Cole dispersion model (one for each of four relaxation regions $\boldsymbol{\alpha}, \boldsymbol{\beta}, \boldsymbol{\gamma}$, and $\boldsymbol{\delta}[10])$ :

$$
\hat{\varepsilon}(\omega)=\varepsilon_{\infty}+\sum_{n=1}^{4} \frac{\varepsilon_{s}-\varepsilon_{\infty}}{1+\left(i \omega \tau_{n}\right)^{\left(1-\alpha_{n}\right)}}+\frac{\sigma_{i}}{i \omega \varepsilon_{0}},
$$

where $\tau_{n}$ are the relaxation times, $\varepsilon_{\infty}$ is the infinite permittivity when $\omega \tau \gg 1, \varepsilon_{s}$ is the static permittivity $(\omega \tau \ll 1), \sigma_{i}$ is the ionic conductivity, and $\alpha_{n}$ are the measures of the dispersion broadening. 


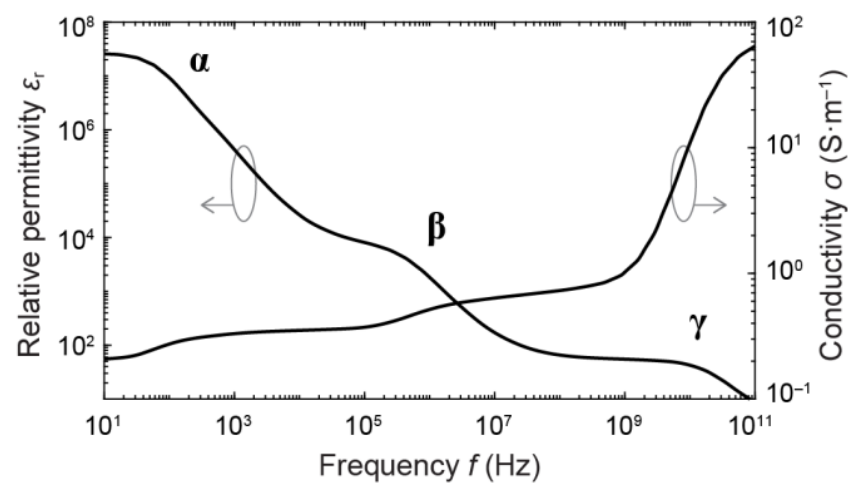

Fig. 1. Electromagnetic properties and relaxation regions of muscle tissue.

Fig. 1 shows the EM properties of muscle modeled using (1) based on experimental data obtained by Gabriel et al. [11]. For the majority of soft biological tissues, $\varepsilon_{r}(\omega)$ is inversely proportional to $\omega$ and $\sigma(\omega)$ is directly proportional to $\omega$. A number of frequency-dependent mechanisms affect the radiation efficiency of in-body antennas [12]: i) the attenuation in tissue due to dielectric and conductive losses, ii) reflection losses due to wave impedance heterogeneity (the most prominent contrast occurs on skin-air boundary), and iii) the physical limitations on the radiation efficiency $\eta$ of electrically small antennas in lossy media [13], [14]. In addition, body cavities and organs may support resonance modes affecting the radiation efficiency [15], [16]. These mechanisms altogether define the optimal operating frequency in terms of maximum achievable radiation efficiency $\eta_{\text {max }}$.

\section{B. Optimal Operating Frequency}

In addition to the aforementioned mechanisms, the radiation efficiency depends on the operating frequency, antenna position and depth in a body, arrangement of surrounding tissues, and polarization [17]. For instance, the optimal frequency for gastrointestinal applications can be derived using realistic human body model (Fig. 2). We consider two point sources that excite $\mathrm{TM}_{10}$ and $\mathrm{TE}_{10}$ modes. The source is bounded by a $\varnothing 4-\mathrm{mm}$ closed surface $C_{S}$ on which the supplied power $P_{s}$ can be evaluated independently of the source formulation as

$$
P_{s}=\oint_{C_{S}}\left(\frac{1}{2} \mathbf{E} \times \mathbf{H}^{*}\right) \cdot \mathrm{d} \mathbf{s} .
$$

The exiting power $P_{\mathrm{e}}$ is evaluated on the contour $C_{\mathrm{FF}}$ located at the distance from the source satisfying the far-field criterion as

$$
P_{e}=\int_{C_{F F}}\left(\frac{1}{2} \mathbf{E} \times \mathbf{H}^{*}\right) \cdot \mathrm{d} \mathbf{s} .
$$

The radiation efficiency is then

$$
\eta_{\max }(\omega) \equiv \frac{P_{e}}{P_{s}} .
$$

The formulated problem was solved numerically using in-house $h p$-FEM solver Agros2D [18]; the details of the solution are given in [12]. Fig. 3 shows the achievable radiation efficiencies of the $\mathrm{TE}_{10}$ mode as a function of depth

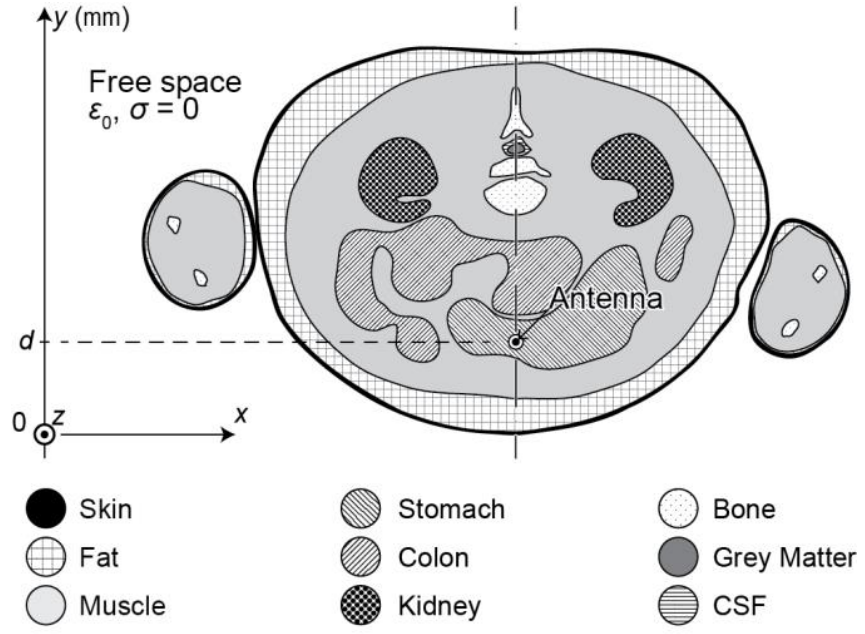

Fig. 2. Optimal operating frequency: problem formulation example for a given application (the body model contains nine dispersive tissues).

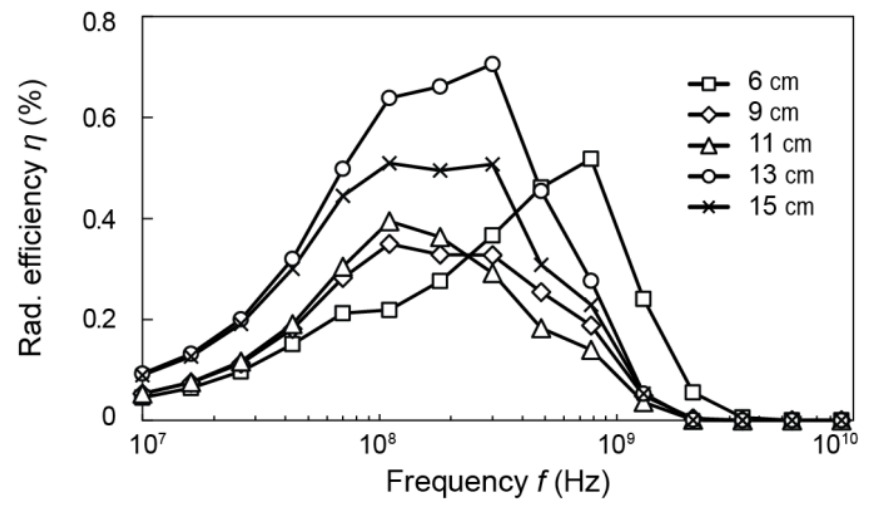

Fig. 3. Achievable radiation efficiency in an anatomical phantom as a function of the source depth.

in the phantom defined in Fig. 2. The efficiency peaks approximately within $200-600 \mathrm{MHz}$ range. Therefore, for gastrointestinal applications the optimal operating bands are MedRadio (Medical Device Radiocommunications Service) (401 to 406,413 to 419,426 to 432,438 to 444,451 to 457) $\mathrm{MHz}$ [19], ISM (Industrial, Scientific and Medical) (433 to 434.8) MHz [20], and WMTS (Wireless Medical Telemetry Service) $611 \mathrm{MHz}$ [21].

\section{Antenna Design Approaches}

A number of in-body antennas have been proposed operating within or close to the aforementioned bands [1], [8], [22]. The first to appear were standard wire antennas adjusted to operate in lossy body tissues. Kwak et al. proposed a helical antenna [23] and Lee et al. reported spiral ones [24], [25]. PCB antennas can achieve better efficiency and robustness then the wire ones [8]. Merli et al. proposed a dual-band (MedRadio $403 \mathrm{MHz}$ and ISM $2.45 \mathrm{GHz}$ bands) multilayer spiral antenna [26] that is fully shielded from the circuitry. Liu et al. reported a wideband $2.45 \mathrm{GHz}$ circularlypolarized multilayer antenna [27] and Dissanayake et al. used the effect of dialectic loading for miniaturization and impedance matching and designed an ultra-wideband (UWB) substrate-integrated slot antenna [28]. The planar printed antennas occupy significant volume (from about $20 \%$ to $50 \%$ ) inside in-body capsules. 
Conformal printed designs, however, occupy negligibly small space: typically less than 5\%. Various types of conformal antennas have been proposed. Izdebski et al. designed a meandered dipole antenna conforming to the interior surface of a capsule [29]. Bao et al. developed a triband inverted-F antenna [30] with the gains $G=-(30,25$, 23) dBi for $403 \mathrm{MHz}, 915 \mathrm{MHz}$, and $2.45 \mathrm{GHz}$, respectively, when simulated inside the stomach of torso model Gustav (CST Microwave Studio [31]).

Loop antennas, in theory, may achieve higher radiation efficiencies than dipoles and monopoles due to their predominantly magnetic near field that couples coupling less to non-magnetic biological tissue [32]. Alrawashdeh et al. proposed a multi-band CSRR-loaded antenna with unprecedented impedance bandwidth [33]. The 434-MHz loop antenna by Suzan et al. [34] extends to the halfspherical extremity of the capsule, therefore increasing the electrical size of the antenna. The main drawback of loop antennas are their sensitivity the variation of EM properties of surrounding tissues (for instance, the resonance frequency of the antenna may shift from $403 \mathrm{MHz}$ in muscle to $4 \mathrm{GHz}$ in air [35]). It requires extreme bandwidths to compensate for the detuning. Therefore, the radiation efficiency has to be sacrificed [36]-[39]. Bao et al., however, developed a workaround to increase the robustness to environment by designing a wideband slot-loop antenna [2].

Microstrip antennas are intrinsically more robust to the varying EM properties of surrounding tissues, as $f_{\text {res }}$ is defined mostly by the substrate geometry and its dielectric properties [40]. However, the microstrip antennas usually have much narrower bandwidths [41] comparing to the loop antennas. Cheng et al. proposed a complementary split-ring resonator (CSRR) loaded $2.45-\mathrm{GHz}$ patch antenna. Mahe et al. employed $\lambda \backslash 4$ stepped impedance resonator along with meandering to miniaturize the microstrip antenna [42]. Recently, we reported a methodology [43] and a miniature 434-MHz antenna design [39] capable of efficient operation from all tissues with high water contents. This approach was further developed in [44] to design a robust dual-band (434 MHz and $2.45 \mathrm{GHz}$ ) antenna.

Despite the progress in antenna design, improving their radiation efficiency remains a major challenge. Efficient numerical modeling and optimization routines facilitate designing antennas that can operate close to the physical bounds on the achievable efficiency for a given application.

\section{ANTENNA MODELING}

We demonstrate the design process using the antenna reported in [39] as an example. This antenna was miniaturized using a $\lambda / 2$ stepped-impedance resonator (SIR) technique and dielectric-loaded with ceramic shell $\left(\mathrm{Al}_{2} \mathrm{O}_{3}\right.$, $\left.\varepsilon_{r} \approx 10\right)$ and pure water inner filling $\left(\varepsilon_{r} \approx 78\right)$. It increases the electrical size of the antenna and therefore reduces the necessary miniaturization up to a factor of $\left(\varepsilon_{r} \text {, eff }\right)^{-1 / 2}$.

The $\lambda / 2$ stepped-impedance resonator can be accurately modeled using the transmission-line-impedance equation (the derivation is given in [42]):

$$
-Z_{1}+Z_{2} \tan \left(\beta_{2} l_{2}\right) \tan \left(\beta_{1} l_{1}\right)=0,
$$

where $Z_{n}(n=1,2)$ are the characteristic impedances of SIR section, $\beta_{n}$ are the phase constants, $l_{n}$ are the section lengths,

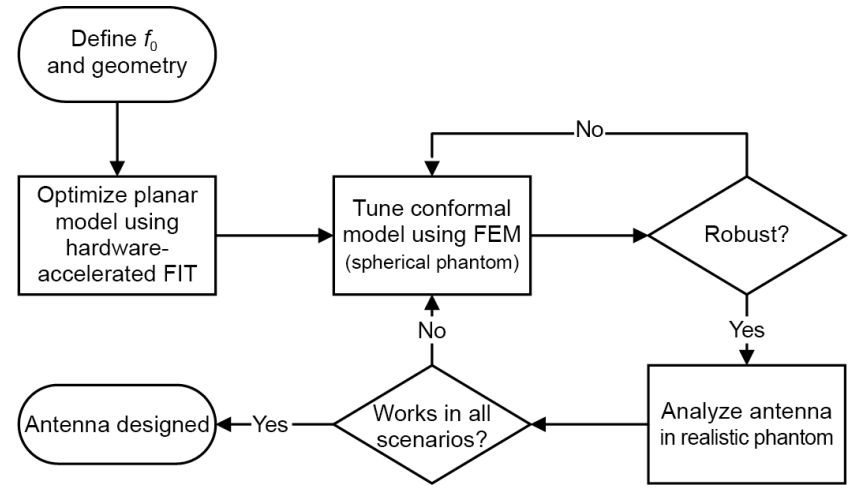

Fig. 4. In-body antenna design flowchart.

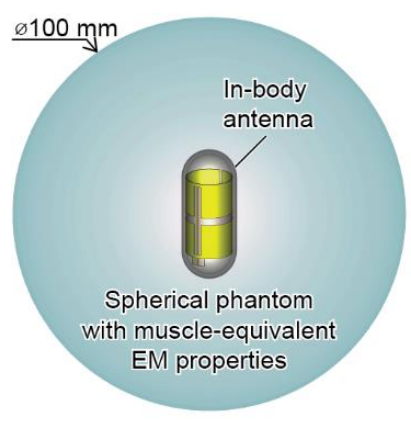

(a)

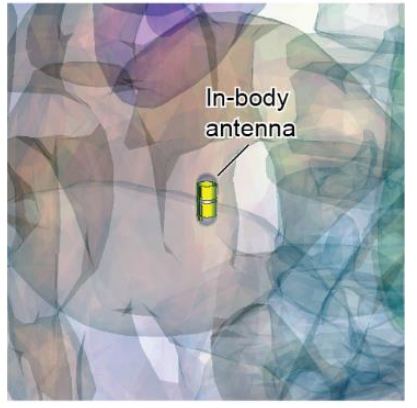

(b)
Fig. 5. Numerical phantoms for antenna analysis and optimization. (a) Canonical $\varnothing 100$-mm spherical homogeneous phantom (not to scale) for antenna analysis and optimization in terms of $\left|S_{11}\right|$ and $\eta$. (b) Fragment of an anatomical phantom used for evaluation of the antenna radiation in realistic application scenarios.

$c$ is the speed of light in vacuum, and $\varepsilon_{r}$, eff are the effective relative permittivities of media surrounding each section.

In this way, one can consider the loading effect of tissue on the antenna already at the analytic stage. After defining the operating frequency and initializing the geometry of the antenna using analytical models, one can proceed to the numerical analysis and optimization of the antenna.

Fig. 4 shows the process flowchart. First, we solved a planar antenna approximation and optimize it in terms of $\left|S_{11}\right|$ using the hardware-accelerated $(2 \times$ NVIDIA Tesla K20c GPUs) TD solver [finite integration technique (FIT)] of CST Microwave Studio [31]. Then, we validate the results using the conformal antenna model in a canonical phantom (Fig. 5a) solved with CST FD solver [finite element method (FEM)]. Both methods used an automatic adaptive mesh refinement ( $\delta\left|S_{11}\right|<1 \%$ for three consecutive passes).

To study the detuning immunity range of the antenna within biological tissues, we analyzed its reflection coefficient $\left|S_{11}\right|$ at $434 \mathrm{MHz}$ in the phantom parametrized to cover the whole range of tissue EM properties (as reported by Gabriel et al. [11]). We defined the range of EM properties as $\varepsilon_{\mathrm{r}} \in[10 . .80], \sigma \in[0 . .2 .4] \mathrm{S} \cdot \mathrm{m}^{-1}$. The antenna design was iteratively adjusted to achieve given robustness to tissues: $\left|S_{11}\right| \leq-10 \mathrm{~dB}$ for all water-based tissues and $\left|S_{11}\right| \leq$ $-3 \mathrm{~dB}$ for infiltrated fat.

For the final antenna, the radiation efficiency and gain values have to be reported for both spherical homogeneous and anatomical phantoms. Spherical symmetry allows for 


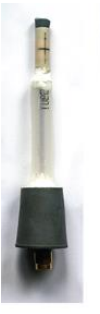

(a)

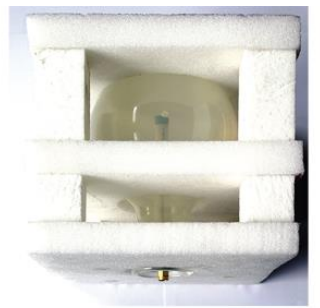

(b)

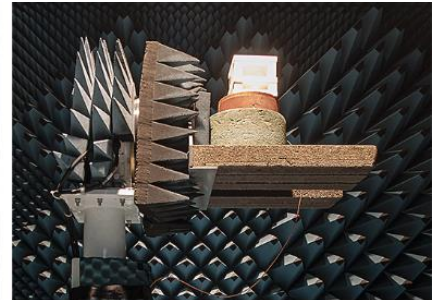

(c)
Fig. 6. Antenna characterization. (a) Antenna prototype; the external feed is insulated with $\varnothing 10-\mathrm{mm}$ polyamide tube. (b) The antenna prototype centered inside of a $100-\mathrm{mm}$ spherical container filled with tissueequivalent liquid. (c) Far-field characterization setup inside of an anechoic chamber.

characterization of radiation performance under wellcontrolled isotropic conditions. It conserves the intrinsic radiation pattern of an in-body antenna and can be reproduced in measurements. In addition, it is a good candidate for a "standard" phantom enabling accurate comparison of radiation performances among different antennas [45].

However, homogeneous phantoms do not give an accurate estimate of the antenna radiation performance for realistic in-body scenarios. For this purpose, we used an anatomically realistic phantom (CST Female Visible Human model "Nelly" optimized for tetrahedral mesh, Fig. 5b) in order to evaluate the capsule radiation performance in different application scenarios.

\section{CHARACTERIZATION}

Radiation characterization of electrically and physically small antennas requires overcoming many challenges. The antenna under test (AuT) couples strongly to a feed that impairs measured data [46]. Radiating currents induced on the feed confound with the antenna radiation. Different approaches have been proposed to mitigate this effect: baluns [47], differential feeds [34], electro-optical converters [48], or measurements using a monostatic scattering characterization of an antenna [49]. For in-body antennas, the problem gets more complicated as the antenna has to be measured in a lossy phantom.

To characterize the antenna prototypes in terms of impedance and radiation, we submerge them into a tissueequivalent liquid. We used a water-sugar-salt [50, Ch. 2] formula to achieve the target $\mathrm{EM}$ properties. The base component is pure water, sucrose $\left(\mathrm{C}_{12} \mathrm{H}_{22} \mathrm{O}_{11}\right)$ reduced the permittivity $\varepsilon_{\mathrm{r}}$, and sodium chloride $(\mathrm{NaCl})$ increased the conductivity $\sigma$. To achieve the target EM properties, we developed a prediction model based on a full factorial experiment along with the response surface optimization methodology [51, Sec. 2.3]. The weighting precision was \pm $0.01 \%$ and the temperature was $25 \pm 0.5^{\circ} \mathrm{C}$. To validate the EM properties, we used the SPEAG DAK kit with DAK-12 probe [52]. To contain the liquid phantom for antenna measurements, we used a $\varnothing 100$-mm spherical glass jar that together constitute the phantom.

We characterize in-body antennas using the direct illumination far-field technique. Inside of the phantom, a 50$\Omega$ coaxial cable links the antenna to an SMA connector that is located outside the spherical jar. A silicone-sealed $10 \mathrm{~mm}$ polyamide tube is fitted between the AuT and the connector to insulate the cable from the phantom (Fig. 6a). The AuT in the phantom constitutes the device under test (DuT, Fig. 6b). The DuT is placed at the distance $d=3 \mathrm{~m}$ from the measurement horn fulfilling the far-field criterion $d \gg 2 \lambda$. An electro-optical converter (enprobe LFA-3 [26]) feeds the DuT. In this way, an optical fiber replaces the RF cable inside the anechoic chamber (Fig. 6c) thus minimizing the error. The estimation of the realized gain uses the gain substitution technique employing a reference antenna of a known gain (ETS-Lindgren Model 3164-06 [27], 300 MHz$\left.6 \mathrm{GHz}, G_{434}=4.5 \mathrm{dBi}\right)$.

\section{CONCLUSION}

In this paper, we presented approaches to in-body antenna design, modeling, and characterization. The modeling approach ensures rapid development of in-body antennas capable of stable operation in a given range of tissues. The established physical bounds on in-body antenna radiation efficiency allow for gauging the radiation efficiency of the final antenna. The accurate link budget evaluation is then possible using the gain evaluation from an anatomical phantom

The characterization approach proposed in here allows for verifying the numerical results and improves the measurement accuracy in two ways. First, spherical phantoms preserve the intrinsic radiation pattern of an antenna. Second, replacing the coaxial feeding cable with an optical fiber and insulating the feed inside of the phantom with air allow suppressing currents induced on the cable.

\section{ACKNOWLEDGMENT}

Results presented in these contributions were developed under the thoughtful guidance of Dr. Maxim Zhadobov, Prof. Ronan Sauleau, and Prof. Pavel Karban. This work was supported in part by the BodyCap Company, in part by the French National Center for Scientific Research and DGA through the PEPS program, and in part by Rennes Metropole through the AES program.

\section{REFERENCES}

[1] A. Kiourti and K. S. Nikita, "A review of in-body biotelemetry devices: implantables, ingestibles, and injectables," IEEE Trans. Biomed. Eng., vol. 64, no. 7, pp. 1422-1430, Jul. 2017

[2] Z. Bao, Y. X. Guo, and R. Mittra, "An ultrawideband conformal capsule antenna with stable impedance matching," IEEE Trans. Antennas Propag., vol. 65, no. 10, pp. 5086-5094, Oct. 2017.

[3] J. Faerber et al., "In vivo characterization of a wireless telemetry module for a capsule endoscopy system utilizing a conformal antenna," IEEE Trans. Biomed. Circuits Syst., vol. 12, no. 1, pp. 95105, Feb. 2017.

[4] M. K. Magill, G. A. Conway, and W. G. Scanlon, "Tissueindependent implantable antenna for in-body communications at 2.36-2.5 GHz," IEEE Trans. Antennas Propag., vol. 65, no. 9, pp. 4406-4417, Sep. 2017

[5] S. Bakogianni and S. Koulouridis, "On the design of miniature MedRadio implantable antennas," IEEE Trans. Antennas Propag., vol. 65 , no. 7, pp. 3447-3455, Jul. 2017.

[6] Y. Tanabe, T. Chang, A. J. Yeh, and A. S. Y. Poon, "A small dualband asymmetric dipole antenna for $13.56 \mathrm{MHz}$ power and $2.45 \mathrm{GHz}$ data transmission," IEEE Antenn. Wireless Propag. Lett., vol. 13, pp. 1120-1123, 2014.

[7] K. S. Nikita, Handbook of Biomedical Telemetry. Hoboken, NJ: John Wiley \& Sons, 2014.

[8] D. Nikolayev, M. Zhadobov, R. Sauleau, and P. Karban, "Antennas for ingestible capsule telemetry," in Advances in Body-Centric Wireless Communication: Applications and State-of-the-Art, London, UK: IET, 2016, pp. 143-186. 
[9] M. H. M. J. Peters G. Stinstra, "Estimation of the electrical conductivity of human tissue," Electromagnetics, vol. 21, no. 7-8, pp. 545-557, Sep. 2001.

[10] S. Gabriel, R. W. Lau, and C. Gabriel, "The dielectric properties of biological tissues: III. Parametric models for the dielectric spectrum of tissues," Phys. Med. Biol., vol. 41, pp. 2271-2293, Nov. 1996.

[11] S. Gabriel, R. W. Lau, and C. Gabriel, "The dielectric properties of biological tissues: II. Measurements in the frequency range $10 \mathrm{~Hz}$ to 20 GHz," Phys. Med. Biol., vol. 41, pp. 2251-2269, Nov. 1996.

[12] D. Nikolayev, M. Zhadobov, P. Karban, and R. Sauleau, "Electromagnetic radiation efficiency of body-implanted devices," Phys. Rev. Applied, vol. 9, no. 2, p. 024033, Feb. 2018.

[13] C. Pfeiffer, "Fundamental efficiency limits for small metallic antennas," IEEE Trans. Antennas Propag., vol. 65, no. 4, pp. 16421650, Apr. 2017.

[14] M. Shahpari and D. V. Thiel, "Fundamental limitations for antenna radiation efficiency," IEEE Trans. Antennas Propag., in press.

[15] A. Hirata, S. Kodera, J. Wang, and O. Fujiwara, "Dominant factors influencing whole-body average SAR due to far-field exposure in whole-body resonance frequency and $\mathrm{GHz}$ regions," Bioelectromagnetics, vol. 28, no. 6, pp. 484-487, Sep. 2007.

[16] D. Nikolayev, "Radio frequency resonances inside a human head," Comput. Probl. Electr. Eng., vol. 3, no. 1, pp. 73-78, 2013.

[17] A. S. Y. Poon, S. O'Driscoll, and T. H. Meng, "Optimal frequency for wireless power transmission into dispersive tissue," IEEE Trans. Antennas Propag., vol. 58, no. 5, pp. 1739-1750, May 2010.

[18] P. Karban, F. Mach, P. Kůs, D. Pánek, and I. Doležel, "Numerical solution of coupled problems using code Agros2D," Computing, vol. 95, no. 1, pp. 381-408, 2013.

[19] "Medical Device Radiocommunications Service (MedRadio)," Federal Communications Commission (FCC). [Online]. Available: https://www.fcc.gov/medical-device-radiocommunications-servicemedradio. [Accessed: 16-Oct-2017].

[20] International Telecommunication Union (ITU). ITU Radio Regulations (article 5), footnotes 5.138, 5.150, and 5.280. Accessed: Feb. 20, 2018. [Online]. Available: https://www.itu.int/net/ITUR/ terrestrial/faq/index.html\#g013.

[21] "Wireless Medical Telemetry Service (WMTS)," Federal Communications Commission (FCC). [Online]. Available: https://www.fcc.gov/wireless/bureau-divisions/broadband-division/ wireless-medical-telemetry-service-wmts. [Accessed: 16-Oct-2017].

[22] A. Kiourti and K. S. Nikita, "Recent advances in implantable antennas for medical telemetry," IEEE Antennas and Propagation Magazine, vol. 54, no. 6, pp. 190-199, Dec. 2012.

[23] S.-I. Kwak, K. Chang, and Y.-J. Yoon, "The helical antenna for the capsule endoscope," in 2005 IEEE Antennas and Propagation Society Int. Symposium, Washington, DC, 2005, vol. 2B, pp. 804-807.

[24] S.-H. Lee et al., "A wideband spiral antenna for ingestible capsule endoscope systems: experimental results in a human phantom and a pig," IEEE Trans. Biomed. Eng., vol. 58, no. 6, pp. 1734-1741, Jun. 2011.

[25] S.-H. Lee, K. Chang, K. J. Kim, and Y.-J. Yoon, "A conical spiral antenna for wideband capsule endoscope system," in IEEE Antennas and Propagation Society International Symposium, 2008. AP-S 2008, San Diego, CA, 2008, pp. 1-4.

[26] F. Merli, L. Bolomey, J. Zurcher, G. Corradini, E. Meurville, and A. K. Skrivervik, "Design, realization and measurements of a miniature antenna for implantable wireless communication systems," IEEE Trans. Antennas Propag., vol. 59, no. 10, pp. 3544-3555, Oct. 2011.

[27] C. Liu, Y.-X. Guo, and S. Xiao, "Circularly polarized helical antenna for ISM-band ingestible capsule endoscope systems," IEEE Trans. Antennas Propag., vol. 62, no. 12, pp. 6027-6039, Dec. 2014.

[28] T. Dissanayake, K. P. Esselle, and M. R. Yuce, "Dielectric loaded impedance matching for wideband implanted antennas," IEEE Trans. Microw. Theory Techn., vol. 57, no. 10, pp. 2480-2487, Oct. 2009.

[29] P. M. Izdebski, H. Rajagopalan, and Y. Rahmat-Samii, "Conformal ingestible capsule antenna: a novel chandelier meandered design," IEEE Trans. Antennas Propag., vol. 57, no. 4, pp. 900-909, Apr. 2009.

[30] Z. Bao, Y. X. Guo, and R. Mittra, "Single-layer dual-/tri-band inverted-F antennas for conformal capsule type of applications," IEEE Trans. Antennas Propag., vol. 65, no. 12, pp. 7257-7265, Sep. 2017.
[31] Computer Simulation Technology AG. CST Microwave Studio. Accessed: Feb. 20, 2018. [Online]. Available: http://www.cst.com.

[32] D. P. Chrissoulidis and J. M. Laheurte, "Radiation from an encapsulated hertz dipole implanted in a human torso model," IEEE Trans. Antennas Propag., vol. 64, no. 12, pp. 4984-4992, Dec. 2016.

[33] R. S. Alrawashdeh, Y. Huang, M. Kod, and A. A. B. Sajak, "A broadband flexible implantable loop antenna with complementary split ring resonators," IEEE Antenn. Wireless Propag. Lett., vol. 14, pp. 1506-1509, 2015.

[34] M. M. Suzan, K. Haneda, C. Icheln, A. Khatun, and K. Takizawa, "An ultrawideband conformal loop antenna for ingestible capsule endoscope system," in Proc. 10 $0^{\text {th }}$ Eur. Conf. on Antennas and Propagation (EuCAP 2016), Davos, Switzerland, 2016, pp. 1-5.

[35] R. Alrawashdeh, Y. Huang, and P. Cao, "A conformal U-shaped loop antenna for biomedical applications," in Proc. $7^{\text {th }}$ Eur. Conf. on Antennas and Propagation (EuCAP 2013), Gothenburg, Sweden, 2013, pp. 157-160.

[36] A. Karlsson, "Physical limitations of antennas in a lossy medium," IEEE Trans. Antennas Propag., vol. 52, no. 8, pp. 2027-2033, Aug. 2004.

[37] J. S. McLean, "A re-examination of the fundamental limits on the radiation Q of electrically small antennas," IEEE Trans. Antennas Propag., vol. 44, no. 5, pp. 672-676, May 1996.

[38] S. R. Best, "Bandwidth and the lower bound on Q for small wideband antennas," in IEEE Antennas and Propagation Society International Symposium 2006, Albuquerque, NM USA, 2006, pp. 647-650.

[39] D. Nikolayev, M. Zhadobov, L. Le Coq, P. Karban, and R. Sauleau, "Robust ultra-miniature capsule antenna for ingestible and implantable applications," IEEE Trans. Antennas Propag., vol. 65, no. 11, pp. 6107-6119, Nov. 2017

[40] D. Nikolayev, M. Zhadobov, R. Sauleau, P.-A. Chapon, E. Blond, and P. Karban, "Decreasing SAR and enhancing transmission of an inbody biotelemetry capsule by reducing the near-field coupling with surrounding tissues," presented at the BioEM 2015, Monterey, CA, 2015, pp. 465-468.

[41] R. Garg, P. Bhartia, I. Bahl, and A. Ittipiboon, Microstrip Antenna Design Handbook. Norwood, MA: Artech House, 2001.

[42] Y. Mahe, A. Chousseaud, M. Brunet, and B. Froppier, "New flexible medical compact antenna: design and analysis," International Journal of Antennas and Propagation, vol. 2012, May 2012.

[43] D. Nikolayev, M. Zhadobov, P. Karban, and R. Sauleau, "434 MHz ISM band antenna for in-body biotelemetry capsules," in Proc. $11^{\text {th }}$ Eur. Conf. on Antennas and Propagation (EuCAP 2017), Paris, France, 2017, pp. 1035-1038.

[44] D. Nikolayev, M. Zhadobov, and R. Sauleau, "An efficient dual-band conformal antenna with stable $50-\Omega$ matching for versatile in-body applications," IEEE Trans. Antennas Propag, in press.

[45] D. Nikolayev, M. Zhadobov, and R. Sauleau, "Impact of tissue electromagnetic properties on radiation performance of in-body antennas," IEEE Antenn. Wireless Propag. Lett, in press.

[46] L. Huitema, C. Delaveaud, and R. D'Errico, "Impedance and radiation measurement methodology for ultra miniature antennas," IEEE Trans. Antennas Propag., vol. 62, no. 7, pp. 3463-3473, Jul. 2014.

[47] S. Pivnenko, J. Zhang, A. Khatun, T. Laitinen, and J. Carlsson, "Characterization of small antennas for hearing aids by several measurement techniques," in Proc. $4^{\text {th }}$ Eur. Conf. on Antennas and Propagation, Barcelona, Spain, 2010, pp. 1-5.

[48] T. H. Loh, D. Cheadle, and L. Rosenfeld, "Radiation pattern measurement of a low-profile wearable antenna using an optical fibre and a solid anthropomorphic phantom," Electronics, vol. 3, no. 3, pp. 462-473, Aug. 2014.

[49] W. Wiesbeck and E. Heidrich, "Wide-band multiport antenna characterization by polarimetric RCS measurements," IEEE Trans. Antennas Propag., vol. 46, no. 3, pp. 341-350, Mar. 1998.

[50] P. S. Hall and Y. Hao, Antennas and Propagation for Body-Centric Wireless Communications, 2nd ed. Norwood, MA: Artech House, 2012.

[51] D. Nikolayev, "Miniature antennas for biomedical applications," Ph.D. Dissertation, University of Rennes 1, Rennes, France, 2017.

[52] Schmid \& Partner Engineering AG. Dielectric Assessment Kit. Accessed: Feb. 20, 2018. [Online]. Available: http://www.speag.com/ products/dak/dielectric-measurements. 